\title{
FICHES BIBLIOGRAPHIQUES
}

\section{Claudine Vuong}

\section{FICHES POUR UNE DOCUMENTATION \\ A L'USAGE DES FORMATEURS.}

Au cours de notre travail, notre souci a été de respecter trois points de vue:

- une idée directrice,

- une méthode de classification,

- une présentation de chaque ouvrage.

\section{L'idée directrice}

Notre projet est de présenter une bibliographie sélectionnée en fonction de l'utilité, ainsi nous avons retenu quelques principes :

- pas de double emploi,

- ne pas citer d'ouvrage dont l'intérêt est limité,

- couvrir l'ensemble des questions que l'on se pose,

- donner une idée de ce qui peut se trouver.

\section{Quelle classification?}

La recherche en didactique n'a pas été le principe directeur, mais plutôt l'usage pour la formation, en fonction d'un certain nombre de problèmes de formation. Nous reprendrons ici les trois orientations de la didactique proposées par Jean-Louis Martinand : 
- l'orientation normative (celle des programmes, des définitions de disciplines),

- l'orientation praticienne (réflexion sur l'enseignement technique et aide à la préparation des activités scolaires),

- l'orientation critique et prospective (ouverture sur d'autres champs, polémique, points de vue critiques).

\section{Présentation des textes eux-mêmes}

Nous présenterons une fiche par ouvrage, comportant les informations suivantes:

- la caractérisation de l'ouvrage,

- la spécificité de l'auteur,

- un commentaire du contenu, mettant en évidence son intérêt particulier,

- les mots-clés.

\section{Remarques:}

Nous avons volontairement écarté toute sélection de manuels scolaires, trop spécialisés en fonction d'une discipline technique donnée.

Nous pouvons cependant remarquer que la tendance actuelles des manuels scolaires est de se présenter sous forme de précis, ce qui est révélateur de la prééminence des normes; ou sous un aspect "outil-méthode-concept", laissant de côté les aspects fondamentaux.

De plus, il est à noter l'existence de nombreux ouvrages édités par les entreprises elles-mêmes sur un thème technique précis.

D'autre part, à la fin des fiches bibliographiques, nous mentionnons les revues et les thèses présentes au L.I.R.E.S.T à Cachan. 


\section{DÉFINITIONS DE PROGRAMMES ET DE DISCIPLINES \\ PROPOSITIONS MÉTHODOLOGIQUES \\ DOCUMENTS PÉDAGOGIQUES}

\section{Technology in the National Curriculum. UNITED KINGDOM: Departement of Education and Science and the Welsh Office.}

Ce recueil officiel rassemble les référentiels et les programmes d'enseignement de la technologie dans le sytème scolaire britannique, depuis l'école maternelle jusqu'à la fin de ce qui correspond au premier cycle en France. Il comporte deux grands chapitres: conception et technologie - technologie informatique.

Chaque partie est constituée par l'énonciation des différentes capacités à acquérir suivant les différents niveaux scolaires, accompagnée d'exemples de tâches correspondantes; suivie par le programme détaillé d'enseignement.

Mots clés : enseignement de la technologie - Royaume Uni - programme référentiel - capacités - objectifs pédagogiques.

1992. Textes de référence. Technologie. SEVRES : Centre International d'Etudes Pédagogiques. 131 p.

Ce texte présente les deux rapports de la C.O.P.R.E.T. (Commission Permanente de Réflexion sur l'Enseignement de la Technologie). La préface est de Lucien GEMINARD: Il s'agit de définir la technologie en tant que discipline de l'enseignement général.

Le rapport I est une réflexion sur l'enseignement de la technologie, dont la finalité, être un élément important de la culture générale, est définie à partir d'objectifs par niveau de formation, de méthodes, de contenus; la formation des professeurs de technologie est aussi abordée.

Le rapport II qui traite de la technologie dans les lycées d'enseignement général, définit les méthodes et les objets d'étude.

Une annexe propose un modèle de programme d'études, cherchant à définir clairement le rôle spécifique de la technologie et celui de la science, autour du concept central: tâche - action - capacité.

Mots-clés : technologie - discipline scolaire - curriculum - contenus - méthodes - objectifs. 


\section{CANONGE, F., DUCEL, R. 1969. La pédagogie devant le progrès tech- nique. PARIS : P.U.F. 212 p. (épuisé)}

Pour prendre en compte l'évolution rapide des techniques, Fernand Canonge et René Ducel, tous deux professeurs à l'E.N.N.A. de Paris, proposent que la formation professionnelle soit éclairée par la pensée technique: qu'elle soit une éducation à la réflexion technique, qu'elle prépare aux changements et au progrès à venir.

Ils donnent des exemples de problèmes pouvant être abordés avec des élèves, des méthodes d'analyse et de synthèse à partir d'un objet technique, de la famille à laquelle il appartient.

Un chapitre est consacré au dessin technique, une des expression de la pensée technique.

Les auteurs mettent aussi l'accent sur l'importance de la motivation des élèves et sur le travail en équipe.

Mots-clés : pédagogie - enseignement technique - pensée technique - objet technique - dessin technique.

\section{DEFORGE, Y. 1985. Technologie et Génétique de l'objet industriel. PARIS : MALOINE.}

Yves Deforge, inpecteur principal de l'enseignement technique, nous propose une étude systématique et rationnelle des techniques, du point de vue des processus, des organisations et des produits.

Pour organiser une nouvelle culture, la culture technique, il prône de nouveaux outils intellectuels permettant de comprendre les objets industriels: la génétique des objets.

C'est une méthodologie d'examen d'un objet prenant en compte ses propres lois d'évolution, ainsi que sa lignée génétique et le système industriel auquel il appartient.

L'ouvrage se termine sur un exposé rapide de différentes thèses d'analyse de l'objet technique et/ou industriel.

Mots-clés : technologie - génétique - objet industriel. 
GEMINARD, L. 1970 : Logique et Technologie. PARIS : DUNOD. 196 p. (épuisé)

Lucien Géminard, Ingénieur des A.M.et Inspecteur général des Sciences et Techniques industrielles, développe, ici, une méthode d'analyse d'un système matériel.

Il définit la relation technologique, et introduit la notion de fonction technique.

Après quelques rappels indispensables de mécanique et de physique, il expose une méthode de hiérarchisation des fonctions techniques. Cette méthode est ensuite illustrée par des exemples concrets, empruntés à la mécanique.

Mots-clés : analyse fonctionnelle - technologie - relation technologique.

\section{JACOMY, B. 1990. Une histoire des techniques. PARIS : SEUIL. 366 p.}

Bruno JACOMY, ingénieur de Arts et métiers, directeur du Musée national des techniques du C.N.A.M. et enseignant à l'université de Compiègne, nous propose une histoire culturelle des techniques.

Son objectif est de faire apparaître les liens qui unissent toutes les traductions concrètes des techniques à la société.

Pour cela, il présente sept tranches d'histoire des techniques, depuis la préhistoire jusqu'à nos jours, suivant un schéma comportant trois volets: technique,

- un panorama sommaire où il brosse les grands traits de l'évolution

- un objet caractéristique,

- un homme qui reflète l'époque, la culture.

L'ouvrage comporte un index, des tableaux chnronologiques et une bibliographie.

Mots-clés : histoire des techniques. 
MOLES, A. 1957 : La création scientifique. GENEVE : RENE KISTER. 237 p. (épuisé)

Abraham .A. Moles analyse les mécanismes de la création scientifique, de l'invention technique et les comportements intellectuels d'un savant "en acte".

Il établit une classification des différents modes de raisonnement, des cheminements du réseau cognitif dans le processus de la création scientifique.

Ce travail aboutit à un inventaire des systèmes infralogiques et les logiques formelles qui constituent les différentes étapes de ce réseau cognitif complexe, dans lequel évolue le chercheur.

Mots-clés : pensée scientifique - raisonnement scientifique.

PONSART, J. 1989. Objectif objet. Démarches de technologie à l'école maternelle et élémentaire. REIMS : C.R.D.P. (Collection Pratiques de Formation) 67 p.

L'auteur propose un ouvrage pédagogique sur l'enseignement de la technologie à l'école maternelle et à l'école élémentaire. Celui-ci se différencie nettement des travaux manuels parce qu'elle fait plus appel à la réflexion qu'à la pratique: il doit constituer un premier apprentissage de la démarche technologique, initier à la rigueur de la réflexion.

J. Ponsart définit des objectifs, propose les activités et la méthode de cet enseignement de la technologie ( produire et étudier), en respectant une progression de l'objet technique au système technique.

Mots clés : école maternelle - école élémentaire - enseignement de la technologie - objet technique. 
POSTIC, M. 1971. Introduction à la pédagogie des enseignements techniques. PARIS : FOUCHER. 78 p. ( épuisé).

Marcel Postic, professeur de psychopédagogie à l'E.N.N.A. de Paris, précise les grands principes pédagogiques nécessaires pour donner aux élèves de l'enseignement technique les moyens de "s'adapter aux situations humaines et professionnelles qui se présenteront à lui".

Il s'adresse aux enseignants, à qui il conseille différentes méthodes pédagogiques en fonction des principes qu'il a énoncés; il propose des exemples pratiques d'activités précises.

Pour rendre l'action pédagogique plus efficace, l'auteur met l'accent sur la nécessité de mettre en évidence des corrélations lors d'une étude technologique, mais aussi sur l'importance de prendre en compte les stratégies des élèves pour résoudre les différents problèmes auxquels ils sont confrontés.

Mots-clés : pédagogie - psychopédagogie - enseignement technique.

\section{QUARANTE, D. 1984. Eléments de design industriel. PARIS : MALOINE. $475 \mathrm{p}$.}

Danielle Quarante, designer professionnel, est responsable de l'enseignement du design industriel à l'université de Compiègne. L'ouvrage qu'elle signe regroupe des textes et des documents à usage pédagogique ou de recherche, utilisés pour son cours.

Une première partie situe ce qu'est le design industriel, par une démarche étiologique, mettant en évidence les différents facteurs d'émergence et d'évolution qui ont contribué à la constitution de cette discipline professionnelle; on trouve aussi une analyse des différents composants esthétiques d'un produit de conception.

La deuxième partie s'appuie sur les principaux supports théoriques qui soustendent l'enseignement du design industriel: théorie de la forme, sémiologie, communication.

Suivent des élément méthodologiques: la description du processus de conception d'un produit, dans toutes ses composantes (ergonomie, marketing, analyse de la valeur,..), intégrant dans la gestion d'un projet la réalité industrielle et professionnelle.

Mots clés : design industriel - théorie de la forme - esthétique - communication - projet industriel - conception - méthodologie . 

383 p.

Cet ouvrage collectif propose une méthode d'analyse chronologique et hiérarchisée de la "vie" d'un produit industriel.

C'est une démarche descendante, de l'ensemble à l'élémentaire. Utilisant une analyse fonctionnelle, elle constitue un cadre d'élaboration d'un produit industriel, appelé projet industriel, décomposé en activités, elles-mêmes comprenant des phases, parmi lesquelles on distingue des tâches.

Une pédagogie d'approche de cette démarche avec des élèves est proposée.

Mots clés : produit industriel - projet industriel - analyse fonctionnelle technologie - pédagogie.

\section{SANDORI, P. 1983: Petite logique des forces - Constructions et machi- nes. PARIS : SEUIL. 230 p.}

Ayant donné des cours de mécanique aux étudiants en architecture de l'université de Toronto, Paul Sandori, lui-même architecte, nous offre ici un lecture très plaisante..

Il tente de rendre les principes de la statique plus compréhensibles et pour cela reprend des expériences historiques. En effet, puisant dans les auteurs du XVI ème siècle, de Stevin à Pascal, en passant par Torricelli, Newton et Galilée, l'auteur s'inspire du raisonnement de ces illustres savants pour proposer une approche plus aisée des principes généraux de la statique.

Cet ouvrage nous fournit donc des exemples simples et quotidiens pour comprendre la statique, mais aussi replace ces fameux principes dans leur contexte historique.

Mots clés : statique - forces - histoire. 
Ce livre a été conçu il y a plus de vingt ans par un groupe de recherche, the Engineering Concepts Curriculum Project, pour tenter de remédier à la situation alarmante, constatée aux Etats Unis, que de moins en moins d'élèves s'intéressaient aux sciences alors que le monde entrait dans l'ère de la technique.

Il nous propose donc d'aborder l'apprentissage des sciences appliquées et des techniques par une méthode amusante, à partir de sujets pertinents et quotidiens, utilisant une approche systémique.

En présentant des exercices de réflexion et d'analyse, ainsi que des projets, les auteurs introduisent progressivement différents concepts: la prise de décision, la modélisation, le feed-back, la stabilité, la conception logique, la dynamique. L'ordinateur est un des sujets étudiés pour sa fonction particulièrement caractéristique de l'évolution des techniques aujourd'hui.

Mots clés : sciences appliquées - techniques - prise de décision - modélisation - feed-back - ordinateur.

à paraître:

CORRIOL, A ., GONET, A.. Une approche du projet pédagogique. PARIS : C.N.D.P. 


\author{
REFLEXION \\ POINTS DE VUE CRITIQUES \\ OUVERTURES SUR D'AUTRES CHAMPS
}

\title{
AL-HASSAN, A.Y., HILL, D.R. 1991. Sciences et techniques en Islam. Une histoire illustrée. PARIS : EDIFRA / PARIS : UNESCO. 300 p.
}

Les auteurs nous invitent à découvrir les sciences et les techniques en Islam grâce à un panorama historique illusté.

Soulignant les abondantes traces de sciences et de techniques préislamiques, ils veulent cependant démontrer l'influence particulièrement positive de l'Islam, moteur d'innovations méconnues.

Des exemples variés et nombreux témoignent des multiples applications techniques et scientifiques qu'on retrouve par la suite transférées en Occident. Le déclin observé des sciences et des techniques en Islam, à partir du XVIIème siècle, trouve son explication dans la perte de la stabilité politique et avec elle celle de la prospérité économique.

Les auteurs nous invitent toutefois à une attitude optimiste, quant au développement scientifique et technique actuel en Islam, compte tenu de la richesse des ressources naturelles et des ressources humaines dans le monde islamique.

Mots-clés : science - technique - Islam - histoire.

\section{AUMONT, B. \& MESNIER, P.M. 1992. $L^{\prime}$ acte d'apprendre. PARIS : P.U.F. 301 p.}

Bernadette Aumont et Pierre-Marie Mesnier, formateurs d'adultes, ont repris dans ce livre leur travail de thèse de doctorat en Sciences de l'Education. Ils se situent dans le champ de la pédagogie et essaient de mettre en évidence les conditions qui permettent de s'approprier un savoir.

Ils font l'hypothèse que l'acte d'apprendre est une composante de deux processus: chercher et entreprendre. Ils essaient d'en déduire un modèle d'apprentissage.

Les deux chercheurs ont particulièrement porté leur attention à trois dispositifs de formation: le chantier, l'usine, les activités de projet en classe de BTS.

Mots clés : apprendre - chercheur - entrepreneur - pédagogie - modèle pédagogique. 
p.

Jean- Marie Auzias, dans cet ouvrage très court, plaide la cause d'une philosophie des techniques, affirmant que la technique a progressé, au cours des siècles, selon des catégories qui sont aussi celles de l'esprit.

Dans un panorama, de l'Antiquité à nos jours, où il analyse les différentes entre la technique et la culture, il constate, en le déplorant, que ces activités ont été séparées de tout temps, voire opposées.

Il décrit l'évolution chronologique des différentes attitudes philosophiques à l'égard de la technique, pour conclure sur la nécessité d'un enseignement de la pensée technique.

Mots-clés : philosophie des techniques - pensée technique - histoire.

COMBARNOUS, M. 1984. Les techniques et la technicité. PARIS : EDITIONS SOCIALES. 270 p. (épuisé)

L'auteur, ingénieur des Arts et Métiers, propose d'associer à l'examen des objets techniques les méthodes de pensée et de cheminement qui ont contribué à leurs réalisations.

Il définit ainsi la technicité, composante indissociable des techniques.

Trois composantes caractérisent la technicité:

- la rationalité technique : la pensée technique

- l'emploi d'engins : les outils

- les spécialisations : les rôles sociaux.

L'auteur montre aussi l'importance des accords entre les hommes et les choses pour caractériser les activités techniques.

mots clés : technique - technicité - pensée technique. 


\section{DEFORGE, Y. 1993. De l'éducation technologique à la culture techni- que. PARIS : E.S.F. Collection Pédagogies. 159 p.}

Dans cet ouvrage, Yves Deforge cherche à circonscrire le problème de la culture technique.

Il la définit comme une réflexion sur un champ de pratiques et de savoirs à l'aide d'outils d'analyse appropriés.

Après un tour d'horizon des différents positions rencontrées vis-à-vis de la culture technique, il prône une attitude qui soit une réflexion sur le technique conduisant à l'action: "une culture technique pour tous".

Il déplore, en faisant un panorama de l'éducation technologique, en France et en Europe, que la culture technique n'ait jamais été clairement définie, et nous invite à mettre la technique sous contrôle d'une éthique.

En annexe, l'auteur nous présente la trame d'un cours de philosophie des techniques.

Mots-clés : éducation technologique - culture technique - ethique - philosophie des techniques.

DUCASSE, P. 1958. Les techniques et le philosophe. PARIS : PUF. 174p. (épuisé)

Dans cet ouvrage philosophique, Pierre Ducassé cherche à connaître les répercussions probables des activités techniques ou scientifiques sur les activités humaines.

Se situant en rupture par rapport à l'opposition traditionnelle entre la philosophie et les techniques, il nous invite à trouver le "sens" des techniques et la manière d'être des techniciens.

L'auteur analyse la variété des différents significations données au mot technique, soulignant la relativité de chacune.

Un chapitre est consacré à la variété des techniciens: ingénieur, expert, technocrate.

Pour la philosophie il s'agit de comprendre les techniques afin de contribuer à la conscience des actes de la technique.

Une appendice est consacrée à l'expert.

Mots clés : philosophie - technique - expert. 
FURIA, D., SERRE, P. -Ch. 1970. Techniques et sociétés. PARIS : ARMAND COLIN. 447 p. (épuisé)

Daniel Furia et Pierre-Charles Serre, tous deux professeurs de l'enseignement supérieur technique, ont conçu un manuel à l'usage des étudiants. Cet ouvrage est construit pour être une synthèse de l'évolution technique, économique et sociale.

Analysant l'évolution des techniques et leurs interactions, sur trois grandes périodes historiques classiques ( de l'Antiquité au XVIII $^{\circ}$ s., le XVIII ${ }^{\circ}$ s. et le $\mathrm{XIX}^{\circ}$ s., le $\mathrm{XX}^{\circ} \mathrm{s}$.), l'étude est sélective, cependant compensée par une bilbiographie, et accompagnée de nombreux documents (citations, schémas, tableaux...) L'évolution du monde géopolitique est aussi abordée: pays socialistes (écrit en 1970), pays en voie de développement.

Chaque chapitre comporte des textes contradictoires, invitant à la discussion.

Mots clés : techniques - organisations sociales - économie - progrès technique.

GIEDION, S. 1980. La mécanisation au pouvoir. PARIS : Centre de Création Industrielle. 591 p.

Cet ouvrage traduit de l'américain (publié en 1948), reprend la presque intégralité de la première édition de "Mechanization takes command", écrit par Siegfried Giedion, architecte et enseignant suisse.

L'auteur s'y est attaché à analyser les effets de la mécanisation sur notre mode de vie.

C'est une étude historique et ethnologique. Cependant, bien qu'une chronologie soit respectée, il n'y a pas accumulation historique de faits, mais mise en évidence de l'évolution des phénomènes techniques sur de longues périodes, à travers les implications quotidiennes et les arts.

Les phénomènes techniques abordés vont de l'artisanat ancien au travail à la chaîne et l'organisation du travail qu'il implique, de l'évolution des "substances organiques" (sol, pain, viande) à celle de l'environnement humain, et en particulier l'idée de confort, les tâches ménagères, le bain.

L'ouvrage est largement illustré et l'auteur conclut à la nécessité de conserver l'équilibre fragile de la vie humaine dans toute ses dimensions: travail, vie quotidienne.

Mots clés : mécanisation - machines - artisanat - organisation du travail vie quotidienne. 
GILLE, B. (sous la dir. de).1978. Histoire des techniques. PARIS : GALLIMARD. Encyclopédie de La Pleïade. 1649 p.

Cet ouvrage monumental a été écrit sous la direction de Bertrand Gilles, celui-ci a cependant rédigé $90 \%$ de l'ensemble.

Le parti pris de cette histoire des techniques est celui de l'histoire des systèmes techniques, de leur chronologie, en cherchant à expliquer par quel progrès technique s'effectue le passage d'un système à l'autre, en s'attachant à montrer que c'est une histoire "enchaînée au monde matériel".

Plus de la moité des articles sont consacrés à une histoire chronologique des techniques depuis la préhistoire jusqu'à nos jours.

Dans une deuxième partie, sont analysés les rapports de la technique avec le monde: l'influence qu'elle subit et qu'elle lui fait subir: on y trouve en particulier un article de Bernard Quémada à propos de Technique et Langage et de Bertrand Gille, Technique et Droit et un Essai sur la connaissance technique.

L'ensemble constitue une source exceptionnelle de renseignements, comprenant des illustrations nombreuses, des tableaux, des graphiques.

L'ouvrage comprend, d'autre part, un tableau synchronique, un index et une table analytique montrant le souci de l'auteur de rendre facile son utilisation.

Mots-clés : histoire des techniques - systèmes technique - progrès technique - technique et langage.

HAUDRICOURT, A.G. 1987. La technologie science humaine. Recherches d'histoire et d'ethnologie des techniques. PARIS : Editions de la Maison des SCIENCES DE L'HOMME. $341 \mathrm{p}$.

André-Georges Haudicourt, est agronome de formation, puis linguiste et technologue; dans cet ouvrage sont rassemblés différents articles qu'il a rédigés, à différentes dates, à propos de la technologie: science humaine. Il situe son action pour une meilleure compréhension de l'homme et des hommes.

En précisant le sujet de la technologie, il insiste sur l'importance des points de vue avec lesquels on peut l'aborder, en tant qu'activité matérielle des populations". Il nous propose une méthodologie, étudiant les aspects historique, géographique, fonctionnel et dynamique des techniques, tout en insistant sur la nécessité d'une étude empirique.

La technique est définie comme une activité "socialement apprise et socialement transmise".

Mots clés : technologie - technique - science humaine - science sociale. 


\section{LAFITTE, J. 1932. Réflexions sur la science des machines. PARIS: : VRIN. 122 p.}

J. Lafitte, ingénieur et architecte, considère qu'il est nécessaire de connaître et comprendre les machines pour mieux appréhender le progrès humain.

Il propose les bases de constitution de la science des machines, qu'il nomme mécanologie. Il définit une organisation en différents types de ces corps organisés par l'homme, que sont les machines:

- machines réflexes,

- machines actives,

- machines passives,

où l'on peut distinguer des séries et les lignées, à partir de critères d'évolution.

Cette science normative des corps organisés et construits par l'homme, est aussi une science sociale puisqu'elle s'appuie sur des critères définis par l'activité humaine.

mots clés : machines - mécanonologie - science des machines.

\section{LE CHATELIER, H. 1947. De la méthode dans les sciences expérimen- tales. PARIS : DUNOD. 319 p. (épuisé)}

Ingénieur des Mines, enseignant, chercheur, Henri Le Chatelier nous livre, avec conviction, les conclusions auxquelles il est parvenu après ses diverses activités scientifiques et de professeur.

Il expose les principes fondamentaux de la méthode scientifique:

observation des faits: recherche de facteurs ;

mesures: degré de précision ;

raisonnement: importance de la logique et du calcul, interpréta tion des mesures ;

lois: déterminisme ( croyance à la nécessité de lois pour prévoir)

Tous ces points sont largement illustrés par des expériences personnelles de l'auteur. Il aborde ensuite les inter-relations entre industrie et science (dont le taylorisme est un exemple) et la nécessité d'un enseignement de la science industrielle (comprenant des.stages en entreprise et des travaux de laboratoire).

Mots clés : méthode scientifique - industrie - faits scientifiques - mesures raisonnement - lois. 
LEON, A. 1965 Formation générale et apprentissage du métier. PARIS : P.U.F. 395 p.

Dans cet ouvrage, l'auteur prend en compte la spécificité de la pensée technique, en analysant, par une démarche psychopédagogique, les rapports entre l'enseignement général et les stages professionnels proposés à des apprentis qui préparent un CAP de mécanique.

Cette analyse, très complète, permet de mesurer l'influence des facteurs psychosociologiques des apprentis scolarisés, ainsi que ceux de leurs maîtres, et de situer les relations entre le niveau scolaire et l'importance des transferts entre les différentes activités professionnelles, de comparer les processus mentaux mis en jeu dans les exercices scolaires et les travaux d'atelier.

Ceci constitue une des premières études attirant l'attention sur le fait que les apprentis construisent des règles d'action, "économiques" par rapport aux tâches demandées,.différentes de celles qu'on attend.

Mots clés : apprentissage - transfert - stages professionnels.

LEROI-GOURHAN, A. 1964. Le geste et la parole. PARIS : A. MICHEL. 2 vol. (1, Technique et langage ; 323 p. 2, La mémoire et les rythmes. 285 p.)

André Leroi-Gourhan, préhistorien et paléontologue, se propose de situer l'homme futur dans son présent et son passé le plus lointain ( la préhistoire). L'auteur circonscrit ses observations aux critères d'humanité: la station verticale qui libère la main pour l'outil, la face courte qui se prépare au langage. Il met en évidence les étroites relations entre les évolutions biologiques qui ont permis l'outil et celles qui ont amené au langage: la technique est à la fois geste et outil, la syntaxe opératoire qui organise leurs relations est comparable au langage.

Le premier volume développe l'analyse des modes d'expression de l'homme jusqu'à nos jours: de la naissance de l'écriture à l'audiovisuel contemporain, en passant par toutes les formes de graphismes.

Dans le deuxième volume, André Leroi-Gourhan s'intéresse à l'opposition entre le matériel et le spirituel et dans un premier temps à la confrontation entre l'instinct et le langage. L'auteur analyse le comportement opératoire de l'homme suivant trois niveaux: le comportement automatisé, le comportement machinal et le comportement lucide. Il développe ensuite les différents stades de régression de la main. L'auteur décrit le comportement esthétique en ce qu'il est caractéristique des relations entre l'individu et le groupe. 
L'homme contemporain apparaît comme le dernier stade d'évolution de l'homo sapiens de la zoologie avec les problèmes que pose la perte de l'activité manuelle puique "un des résultats l'étude simultanée de l'homme sous les angles de la biologie et de l'ethnologie est de montrer le caractère inséparable de l'activité motrice .. et de l'activité verbale".

Mots-clés : anthropologie - paléontologie - technique - geste - langage outil - comportement opératoire - esthétique.

\section{LEROI-GOURHAN, A. 1992. (1ère édition 1943). L'homme et la ma- tière. PARIS : A. MICHEL. 326 p.}

Préhistorien et paléontologue, André Leroi-Gourhan explore le tissu des relations que l'homme a maitrisé entre lui et la nature, c'est-à-dire la technologie, seule activité humaine qui montre une totale continuité dans le temps.

La méthode choisie est de collecter des faits techniques, de les observer sur plusieurs groupes humains et d'en faire une confrontation, afin de discerner la succession des stades techniques et confirmer les grandes lignes de constructions logiques: établir une hiérarchie des techniques. Cette étude est conduite à partir d'observations préhistoriques et ethnologiques. La méthode est, en elle-même, pleine d'enseignement puisqu'elle permet d'échapper à l'analyse des seules tendances.

L'édition de 1992, postérieure à la parution de " Le geste et la parole", permet à l'auteur d'établir des liens entre entre les deux ouvrages.

Mots -clés : ethnologie - technique.

LOJKINE, J. 1992. La révolution informationnelle. PARIS : P.U.F. Sociologie d'aujourd'hui. 297 p.

Jean Lojkine, agrégé de philosophie et sociologue, analyse dans cet ouvrage la mutation du monde économique. Plusieurs explications sont envisagées, selon les théories écomomiques en vigueur: américaine (TaylorismeFordisme) et japonaise (Kan-ban). L'auteur démontre le poids de la tradition et propose la vision nouvelle d'une révolution informationnelle. Il essaie de dépasser les critères traditionnels de gestion, dont il analyse les insuffisances: divisions hiérarchiques, linéarisation de l'information, non prise en compte de la cybernétique.

Ayant mis en évidence l'enjeu de la maîtrise de l'information, Jean Lojkine invite à changer de règle du jeu, à dépasser le modèle linéaire et hiérarchique qui régit le monde technologique en particulier, la société en général. 
Mots clés : révolution industrielle - information - cybernétique - économie. MALGLAIVE, G. 1990. Enseigner à des adultes. PARIS : P.U.F. 285 p.

Gérard Malglaive est directeur du centre de formation de formateurs du C.N.A.M.

A la lumière de son expérience de formateur d'adultes, il se propose, dans ce livre, d'analyser comment les adultes accèdent aux connaissances formalisées à partir de la confrontation à la seule pratique: les activités pratiques sont instrument et finalité de la formation.

L'auteur s'engage à montrer le rôle fondamental de l'action dans la construction des savoirs, éclairant son propos par des modèles théoriques: analyse du travail, analyse du savoir, objectifs pédagogiques, psychologie cognitive.

S'attardant ensuite sur quelques exemples illustratifs, il termine par des propositions de dispositifs pédagogiques concrets.

Mots clés : enseigner - formation d'adultes - apprentissage - cognition - pédagogie.

\section{MARTINAND, J.L. 1986. Connaître et tranformer la matière. GENEVE : Peter LANG. 315 p.}

Jean-Louis Martinand, physicien, professeur à l'Ecole Normale Supérieure de Cachan, a rassemblé, dans ce livre, différentes propositions pour l'enseignement des Sciences Physiques et de la Technologie, à partir de son expérience, en particulier celle d'avoir participer à la commission "Lagarrigue".

L'auteur s'est attaché à caractériser les objectifs de l'enseignement et son analyse fait apparaître la notion d'objectif-obstacle, ainsi que la nécessité de cohérence entre objectifs, finalités contenus, activités et démarches. Il développe cette théorie en décrivant trois innovations:

initiation aux techniques de fabrication mécaniques, à propos de laquelle il introduit la notion de pratique sociale de référence,

concept d'élément chimique,

notion de dureté.

L'auteur trace ensuite les perspectives pour l'enseignement et la recherche.

Mots clés : didactique - technologie - sciences physiques - fabrication mécanique - élément chimique - dureté - objectif obstacle - pratique de référence. 
MUMFORD, L. 1950. Technique et civilisation. PARIS : SEUIL Collection Esprit. 415 p.

L'auteur analyse les modifications de la culture de la civilisation occidentale sous l'influence du développement du machinisme ;

Il propose de classer le développement de la machine et de la civilisation machiniste en trois phases:

- la phase éotechnique

- la phase paléotechnique

- la phase néotechnique.

Elles représentent chacune une période de l'histoire humaine, caractérisées par ce qu'elle forment un complexe technologique, fait de ressouces et matières premières, de formes de production, de types d'ouvriers, de méthode d'éducation des ouvriers et d'un développement de certaines aptitudes.

Mots clés : machine - technique - civilisation

PELPEL , P., TROGER, V. 1993. Histoire de l'enseignement technique. PARIS : HACHETTE. 319 p.

Patrice Pelpel, agrégé de philosophie, ancien formateur au C.F.P.E.T. de Cachan et Vincent Troger, docteur en histoire, nous offre une histoire de l'enseignement technique construite en quatre grandes parties, illustrée par de nombreux tableaux statistiques et des extraits de textes officiels ou d'ouvrages.

La première partie montre comment s'est constituée cette spécificité française qu'est l'enseignement technique et professionnel- ce modèle français-, à partir de la nécessité de structures d'apprentissage, d'abord d'initiatives privées, auxquelles se sont substitués, par les interventions de l'Etat, un ensemble d'établissements d'enseignement.

Dans un deuxième temps, les auteurs précisent tous les éléments qui ont abouti à l'intégration de l'enseignement technique au sein d'un système éducatif unifié.

Une troisième partie est consacrée à l'étude des profils sociaux et pédagogiques des élèves et des professeurs du technique.

La dernière partie tente de définir la culture technique sous tendue par les différentes réalités de l'enseignement technique et de témoigner de la diversité des activités pédagogiques constatées, souvent innovantes.

Mots clés : histoire - enseignement technique - apprentissage - culture technique - pédagogie. 
PRADES, J. et al. 1992. La technoscience- Les fractures du discours-. PARIS : L'HARMATTAN. 288p.

Jacques Prades, rassemble dans cet ouvrage récent les thèses de différents intervenants sur "la question de la technique", et plus précisément sur la "technoscience".

Les questions se posent de la même manière qu'il y a quelques années: "D'où vient-elle?" et "Qu'est-ce que cela change?", mais depuis la contribution décisive de G. Simondon (1958) entre autres, l'histoire a déjà donné des réponses et d'autres opinions se sont fait connaître.

Il est particulièrement intéressant de faire le point sur les nouvelles réponses à ces questions, éclairées par des points de vue aussi divers que la sociologie, l'économie, la philosophie et la psychanalyse.

Ce livre polémique nous propose une réflexion nouvelle, alimentée par les opinions les plus contradictoires sur la technique, la science et leurs implications dans la société.

Mots-clés : technoscience - économie- sociologie.

\section{ROQUEPLO, P. 1983. Penser la technique. Pour une démocratie concrète. PARIS : SEUIL. 249 p.}

Philippe Roqueplo a enseigné la philosophie des sciences; dans cet ouvrage, il rassemble une série de contributions, datant de 1974 à 1982, dans lesquels il démontre que la technique appartient au terrain de l'ethnologie, et donc ne saurait être considérée hors de la société et des problèmes sociaux et humains.

Il analyse les différentes implications des techniques sur les plan épistémologique, éthique, sociologique, politique et pédagogique.

Il insiste sur la nécessité de négocier démocratiquement les changements technologiques, et donc de promouvoir une culture technique.

Mots-clés : technique - philosophie - culture technique . 


\section{RUSSO, F. 1986: Introduction à l'histoire des techniques. PARIS : BLANCHARD. 535 p.}

François Russo nous propose un outil d'analyse de l'histoire des techniques.

Cet ouvrage se veut une grille de lecture des publications concernant l'histoire des techniques, auxquelles l'auteur reproche d'être trop linéaires. François Russo entreprend donc une tâche de restructuration de cette histoire, en prenant des points de vue différents de la chronologie: mettant l'accent sur la dualité présente entre procédé et résultat, il nous présente les grands domaines de la technique.

Mots -clés : histoire des techniques - techniques - domaines techniques.

SIMONDON, G. 1989 (1ère édition 1958) : Du mode d'existence des objets techniques. PARIS : AUBIER. 333p.

Dans cet ouvrage, qui fait référence, Gilbert Simondon, philosophe, développe des arguments pour que la pensée technique soit une catégorie à part entière de la philosophie. Il se place résolument dans une démarche systémique.

Dans une première partie, il analyse l'objet technique dans son évolution ( genèse), pour mettre en évidence le processus de concrétisation- l'objet technique est inventé, pensé, voulu-, et met l'accent sur sa cohérence.

Dans une deuxième partie, l'auteur s'attache aux liens de l'objet technique à l'homme. Il y définit deux catégories: selon le statut de minorité ( l'apprenti) et celui de majorité ( l'ingénieur). Pour mieux analyser la cohérence interne $\mathrm{du}$ fonctionnement de l'objet technique, il propose, dans une troisième partie, une méthode génétique d'examen de la technicité, en tant que mode de relation de l'homme au monde.

L'édition de 1989 comporte une postface de Yves Deforge.

Mots- clés : objet technique - système - concrétisation - technicité - pensée technique. 
1991. Culture technique et formation. Actes du colloque organisé par l'A.E.C.S.E. NANCY : PRESSES UNIVERSITAIRES DE NANCY. $467 \mathrm{p}$.

Cet ouvrage rassemble les différents points de vue de chercheurs ou enseignants sur la culture technique, l'intention du colloque étant de contribuer à ce que la culture technique soit scolairement mieux pensée.

On trouve trois orientations:

- une approche historique et anthropologique des objets techniques,

- une réflexion sur les objets culturels communs au monde de l'entreprise et à celui de l'enseignement,

- la médiation et les techniques.

Chaque contribution est courte, son thème est bien indiqué et répertorié.

Mots-clés : culture technique - enseignement technique - formation technique - qualification - espaces éducatifs - techniques de formation.

REVUES ET THÈSES PRESENTÉES AU L.I.R.E.S.T-CACHAN

REVUES:

Culture Technique.

International Journal of Technology an Design Education.

Publications de Montlignon.

Recherches en didactique des mathématiques.

Technical Education \& Training Abstracts.

Technology \& Culture.

Travail Humain.

THESE:

GINESTIE, J. 1992. Thèse de Doctorat "nouveau régime": Contribution à la didactique des disciplines technologiques. Acquisition et utilisation d'un langage d'automatisme. Université de Provence:. Aix-Marseille I. 